\title{
ENSEÑAR PARA LA COMPRENSIÓN DE FENÓMENOS FÍSICOS Y QUÍMICOS EN LA RESIDENCIA DOCENTE
}

\author{
LUCERO, Irene ${ }^{1}$; GHIONE, Lucrecia ${ }^{2}$; CABANILLAS MENDOZA, Cintia ${ }^{3}$; \\ GILABERT, Romina ${ }^{3}$; BRIZUELA, Miguel Angel ${ }^{4}$
}

Palabras clave: Actividades, Desempeños de comprensión, Enseñanza de la Física y la Química, Residencia docente.

\section{Resumen:}

Planificar las situaciones de enseñanza-aprendizaje supone tomar decisiones vinculadas al qué, cómo y con qué enseñar y evaluar. El desafío más grande con el que se encuentra el futuro profesor en las prácticas de residencia es el diseño de las clases, no sólo para motivar, sino para que los alumnos comprendan los fenómenos físicos o químicos que se están trabajando en el aula de ciencias. En este trabajo se muestran algunas secuencias de actividades usadas por practicantes de los profesorados de Física y de Química, en aulas de escuelas secundarias. Se presenta el análisis de las consignas, desde la enseñanza para la comprensión, discriminando los desempeños de comprensión de la física o la química presentes en cada actividad. Este análisis es considerado relevante porque hace tomar conciencia al profesor de qué se espera del alumno para asegurarnos que aprende, asumiendo que aprender en ciencias naturales es comprender el modelo involucrado para explicar un determinado fenómeno.

\section{INTRODUCCIÓN:}

El concepto de práctica docente es un concepto complejo y de múltiples dimensiones que abarca aspectos disciplinares, epistemológicos, sociales, históricos, políticos, pedagógicos y didácticos.

Formarse en las prácticas docentes es un proceso permanente, que acompaña toda la vida profesional, pero es en la formación inicial y, sobre todo, en las prácticas de enseñanza, donde los estudiantes adquieren $y$ desarrollan los cimientos fundamentales de la profesión. (Davini, 2015,p42).

1. Prof. en Matemática, Física y Cosmografía, Magister en Metodología de la Investigación Científica y Técnica- Departamento de Física, FaCENA.TE: 379-4546856. irmaireneprof@gmail.com

2. Prof. en Ciencias Químicas y del Ambiente. Bioquímica. Departamento de Humanidades. FaCENA. TE: 3794673754.lucreciaghione@yahoo.com.ar

3. Prof. en Física. Cátedra Didáctica de la Física y Práctica de Residencia.FaCENA. cintiacabanillasmendoza@gmail.com

4. Alumna Prof. en Física. Cátedra Didáctica de la Física y Práctica de Residencia. FaCENA. omigilabert@gmail.com

5. Alumno Prof. en Cs. Químicas y del Ambiente. Cátedra Didáctica de la Química y Práctica de Residencia. FaCENA 
Didáctica de la Física y Práctica de Residencia en el Profesorado en Física y Didáctica de la Química y Práctica de Residencia en el Profesorado en Ciencias Químicas de la FaCENA (UNNE), son asignaturas anuales del cuarto año y, desde el punto de vista curricular, constituyen el último peldaño del trayecto de formación pedagógica del profesor. Podría entonces entenderse que la Práctica de Residencia se constituye en un apéndice de la formación teórica previa (Sanjurjo y otros, 2004). Sin embargo, estas asignaturas se desarrollan como espacios de articulación y convergencia de los conocimientos construidos a lo largo de la carrera en los otros trayectos de formación, donde el ejercicio de la práctica y la lectura de la misma se conjugan en un todo armónico, que involucra a los conocimientos disciplinares y a los pedagógico-didácticos.

Si bien para el residente el campo educativo no le resulta desconocido, en virtud de que en las diferentes asignaturas del trayecto pedagógico ya ha incursionado en el mismo, es en el espacio de práctica de residencia donde se consolida el verdadero ejercicio de la enseñanza, desde el qué enseñar hasta la evaluación de los aprendizajes. Sanjurjo y Foresi (2015) citando a Shulman distinguen siete tipos de conocimientos que el docente debe manejar para resolver las situaciones de enseñanza: del contenido científico, pedagógico, del currículo, de los alumnos y el aprendizaje, del contexto, de filosofía y el conocimiento didáctico del contenido. Este último es el que cobra más importancia al momento de la práctica docente en las escuelas, dado que, "se entiende por conocimiento didáctico del contenido a la forma en que el docente procesa el contenido a enseñar, de tal manera que el mismo se transforme en contenido enseñable para que los estudiantes lo comprendan, sin que el mismo sea deformado". (Sanjuro y Foresi, 2015,p29). Esto obliga a que el que enseña no sólo debe saber el conocimiento disciplinar sino también las dificultades para comprender ese contenido disciplinar y cómo hacer para resolver esas dificultades.

Como profesores de Física y Química es importante tener en claro ¿qué significa saber un contenido de física o química?, ¿Cómo se da cuenta el docente que el alumno comprende un concepto físico-químico o una ley determinada? Para dar respuestas a estas preguntas se tomaron como marco los lineamientos de la teoría de la enseñanza para la comprensión de Perkins (1999). Esta teoría subraya cuatro conceptos claves:

Tópicos o temas generativos: se refiere al contenido seleccionado para enseñar, desde la centralidad que él representa en el listado de temas.

Metas de comprensión: que se refiere a lo importante dentro del tema; guía qué es lo que se quiere que aprendan y por ello puede vincularse con los objetivos planteados para la clase

Desempeños de comprensión: se refiere a lo que deben hacer los estudiantes y que hay que pedirles que hagan; tienen estrecha relación con las actividades que se propongan. 
Evaluación continua: se refiere al seguimiento para registrar y saber lo que han comprendido, una evaluación para la retroalimentación, teniendo el estudiante oportunidades para reflexionar sobre sus propios aprendizajes.

De estos cuatro conceptos el que cobra relevancia es el de los desempeños de comprensión. Perkins utiliza el criterio de desempeño flexible para entender al aprendizaje: "comprender un tópico quiere decir ni más ni menos que ser capaz de desempeñarse flexiblemente en relación con el tópico: explicar, justificar, extrapolar, vincular $y$ aplicar de maneras que van más allá del conocimiento y la habilidad rutinaria". Si el alumno es capaz de realizar en forma autónoma distintos desempeños que el docente considera como relevantes dentro de la disciplina que enseña, se puede decir que comprende el contenido. Entonces, en Física y Química ¿cuáles serían los desempeños de comprensión específicos?; asumiendo que la Física y la Química explican a la naturaleza usando modelos, estos desempeños serían: representar esquemáticamente el sistema en estudio, identificar las variables que describen a un fenómeno, relacionar esas variables, interpretar ecuaciones químicas y expresiones matemáticas de leyes, utilizar la expresión de una ley para analizar los fenómenos estudiados, realizar mediciones, tabular datos, graficar e interpretar gráficas, predecir, explicar y argumentar el comportamiento de un sistema, nombrar compuestos químicos, escribir correctamente la nomenclatura química. Estos son desempeños importan- tes que deben ser desarrollados por medio de las actividades de aprendizaje que se propongan en las clases.

En este trabajo se presentan las secuencias de actividades diseñadas y aplicadas por residentes de los Profesorados de Física y de Química en los años 2016 y 2017 de la FaCENA, para sus prácticas en las escuelas secundarias; se muestra el análisis didáctico realizado a las mismas, desde los desempeños de comprensión que promueven y reflexiones finales sobre esta postura a la hora de planificar y el desempeño de los practicantes en esta instancia.

La implementación de las secuencias en las aulas es parte del trabajo de campo que se lleva a cabo dentro del proyecto de investigación y desarrollo PI 17/12 F005 "Universidad y Escuela Secundaria mancomunadamente por la Enseñanza Aprendizaje de la Física", subsidiado por la Secretaría de Ciencia y Técnica de la UNNE.

\section{DESCRIPCION DE LA PRO- PUESTA}

La inserción de los estudiantes en la realidad áulica donde efectivamente realizan la práctica profesional se va dando en forma gradual. Con la lógica y la metodología de la investigación científica del campo social, se busca aproximarlos a la realidad de los distintos momentos del proceso enseñanza- aprendizaje, buscando información para ser analizada críticamente a la luz de los marcos teóricos que se desarrollan a lo largo del año. 
Se da entonces la inmersión de los estudiantes de una manera guiada por el profesor de práctica y los docentes colaboradores (tutores de formación docente) de las escuelas que los reciben. Se inician con una ronda de observaciones en distintas aulas y establecimientos escolares, que luego se transforman en un período de pasantía integrada al grupo en el que hará la residencia; esta pasantía tiene dos momentos: el inicial donde el residente sólo es un observador no participante que recoge datos para el análisis del proceso de enseñanza y adentrarse en la modalidad de trabajo del aula de práctica y otro posterior, donde colabora en las actividades docentes en el aula con la guía activa del profesor tutor y el constante apoyo y evaluación del profesor de práctica, iniciándose en la programación de actividades áulicas y el apoyo a la enseñanza.

Terminado el período de pasantía, el residente se hace cargo del desarrollo de las clases, enseñando los contenidos propuestos por el profesor tutor, cumpliendo con la planificación de las clases, la enseñanza y la evaluación de los aprendizajes de los estudiantes. En la etapa de la planificación de las clases se da una relación muy personalizada entre el residente y el profesor de práctica, que realiza un seguimiento pormenorizado al practicante. Se llevan a cabo instancias de reflexión y discusiones entre pares y con el docente de práctica, respecto de las actividades áulicas que se diseñan para el aprendizaje de los contenidos. Es justamente en esta etapa, donde se requiere que el residente realice el análisis de cada una de las actividades propuestas, bajo la lupa de los desempeños de comprensión que se promueven con cada una de ellas.

En las tablas siguientes se muestran algunas de las secuencias de actividades, diseñadas por dos residentes que han hecho las prácticas en cursos del ciclo orientado de la educación secundaria en escuelas públicas.

\section{$5^{\circ}$ año}

Contenidos: Fuerzas- sistema de fuerzas- $1^{a}$ ley de Newton-Primera Ley de Newton

Objetivos específicos

- Interpretar a la fuerza como magnitud vectorial

- Representar fuerzas utilizando escalas

- Distinguir componentes y resultante de un sistema de fuerzas

- Trabajar experimentalmente para medir peso de distintos cuerpos

- Comunicar en forma escrita los resultados de trabajos experimentales sencillos

- Interpretar la primera ley de Newton 
TABLA 1- Fuerzas- sistema de fuerzas- 1a ley de Newton-Primera Ley de Newton

\begin{tabular}{|c|c|}
\hline CONSIGNAS & DESEMPEÑOS DE COMPRENSIÓN \\
\hline \multicolumn{2}{|c|}{ Clase $\mathrm{N}^{\circ} 1$ : FUERZA } \\
\hline $\begin{array}{l}\text { 1. ¿Cuáles son los efectos que produce una fuerza? Dé un } \\
\text { ejemplo en el que por acción de una fuerza un cuerpo } \\
\text { se deforma. }\end{array}$ & $\begin{array}{l}\text { - Identificar el concepto de fuerza } \\
\text { - } \quad \text { Identificar e interpretar los efectos de una fuerza } \\
\text { Aplicar el concepto de fuerza a situaciones } \\
\text { nuevas, ejemplificar }\end{array}$ \\
\hline $\begin{array}{l}\text { 2. En la figura se ven } 2 \text { grupos A y B, compuestos de } 3 \\
\text { personas cada uno que participan de una cinchada. El } \\
\text { equipo A tira hacia la izquierda con una fuerza de } 50 \mathrm{~N} \\
\text { cada uno y el equipo B tira hacia la derecha con una } \\
\text { fuerza de } 40 \mathrm{~N} \text { cada uno. } \\
\text { a) ¿Qué equipo ganará? ¿Por qué? } \\
\text { b) ¿Qué debería pasar para que haya un empate? } \\
\text { c) Representar gráficamente las dos situaciones del } \\
\text { problema, a escala, mostrando todas las fuerzas } \\
\text { involucradas. } \\
\text { Fuente de la imagen: } \\
\text { http://lewiscarroll.files.wordpress.com/2009/06/tensar-la- } \\
\text { cuerda.jpg }\end{array}$ & $\begin{array}{l}\text { - Identificar todas las fuerzas involucradas en un } \\
\text { sistema } \\
\text { - Identificar dirección y sentido que tendrá la } \\
\text { fuerza resultante } \\
\text { - Aplicar el concepto de fuerzas en equilibrio } \\
\text { - Representar las fuerzas como vector } \\
\text { - Usar correctamente la escala }\end{array}$ \\
\hline $\begin{array}{l}\text { 3. Se aplican } 2 \text { fuerzas sobre un bloque en la misma } \\
\text { dirección y sentidos opuestos, es decir } \mathrm{F}_{1}=-15 \mathrm{~N}_{\text {y }} \mathrm{F}_{2}= \\
25 \mathrm{~N} \text {. } \\
\text { a) Halle la resultante } \\
\text { b) Dé las características de la resultante. }\end{array}$ & $\begin{array}{l}\text { - } \quad \text { Elegir una escala adecuada } \\
\text { - } \\
\text { - Cacer el trazado de fuerzas componentes y } \\
\text { - Caracterizar la fuerza resultante } \\
\end{array}$ \\
\hline $\begin{array}{l}\text { 4. Dada las fuerzas } \mathrm{F}_{1}=40 \mathrm{~N} \text { y } \mathrm{F}_{2}=30 \mathrm{~N} \text {, ángulo } 120^{\circ} \\
\text { a) De una escala adecuada para representar. } \\
\text { b) Halla la resultante de las fuerzas gráficamente y } \\
\text { exprese su valor aproximado, según la escala } \\
\text { elegida. } \\
\text { c) Diga el método que utilizó. }\end{array}$ & $\begin{array}{ll}\text { - } & \text { Interpretar escala } \\
\text { - } & \text { Hacer el trazado de fuerzas componentes y } \\
\text { - } & \text { resultante } \\
\text { - Graficar la fuerza resultante }\end{array}$ \\
\hline
\end{tabular}

\section{Clase N²: PRIMERA LEY DE NEWTON}

1. Discutan si la siguiente afirmación está de acuerdo con $\quad$ - Interpretar el significado del primer principio el primer principio de la dinámica: "Para mover un cuerpo se necesita siempre una fuerza". Justificar.

2. En los siguientes casos decir quién tiene mayor inercia y por qué.

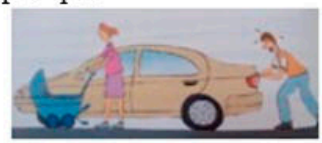

(a)

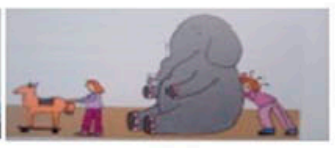

(b)
- Justificar explicaciones usando conceptos

Identificar quién tiene mayor inercia en las imágenes observadas

- Vincular inercia con el concepto de masa

- Explicar de manera fundamentada la inercia

- Justificar explicaciones usando conceptos

(Fuente de la imagen: Santillana Perspectiva. 2007)

\section{Clase No 3: LABORATORIO: PESO}

Se quiere saber el peso de los objetos suministrados

1. ¿Qué instrumento de medición utilizaremos? Sacar una
Conocer las partes y aprender a usar un instrumento de medición (dinamómetro) 


\section{Clase No 3: LABORATORIO: PESO}

Se quiere saber el peso de los objetos suministrados

1. ¿Qué instrumento de medición utilizaremos? Sacar una foto o dibujar y colocar el nombre de sus partes. Explicar el funcionamiento.

2. ¿Cuál es la precisión del instrumento?

3. Elabora una tabla con los valores medidos.

4. Realice el informe describiendo el trabajo realizado.
- Conocer las partes y aprender a usar un instrumento de medición (dinamómetro)

- Identificar la magnitud peso (fuerza), que se va a medir.

- Observar la escala del instrumento

- Identificar la mínima lectura del instrumento

- Expresar correctamente una medición

- Aplicar conceptos de precisión

- Comunicar resultados de trabajo experimental

\section{$6^{\circ}$ año}

Contenidos: Corriente eléctrica- resistividad- Ley de Ohm

\section{Objetivos específicos}

- Definir corriente eléctrica conceptual y matemáticamente

- Caracterizar a los materiales en aislantes y conductores eléctricos

- Manejar algebraicamente las unidades de carga y corriente eléctrica
- Diferenciar conceptualmente los conceptos de resistencia eléctrica y resistividad

- Reconocer los parámetros que afectan a la resistencia de un conductor óhmico

- Determinar experimentalmente la resistencia de un conductor aplicando la Ley de $\mathrm{Ohm}$

- Comunicar en forma escrita los resultados de trabajos experimentales sencillos

TABLA 2- Corriente eléctrica- resistividad- Ley de Ohm

\begin{tabular}{|c|c|c|}
\hline \multicolumn{2}{|r|}{ CONSIGNAS } & DESEMPEÑOS DE COMPRENSIÓN \\
\hline \multicolumn{3}{|c|}{ Clase No 1 : LABORATORIO VIRTUAL } \\
\hline 1 & $\begin{array}{l}\text { 1.Trabajando con la simulación sobre } \\
\text { conductividad eléctrica, dada en la página, } \\
\text { clasifique los materiales que presenta } \\
\text { http://labovirtual.blogspot.com.ar/search/label/c } \\
\text { onductividad\%20el\%C3\%A9ctrica } \\
\text { 2. Elabore un informe de la experiencia } \\
\text { realizada. }\end{array}$ & $\begin{array}{l}\text { - Manipular dispositivo experimental en } \\
\text { entorno virtual } \\
\text { - Inferir características de los materiales } \\
\text { - Registrar datos obtenidos } \\
\text { - } \begin{array}{l}\text { Redactar procedimiento de experiencias } \\
\text { - Comunicar resultados de trabajo } \\
\text { experimental }\end{array}\end{array}$ \\
\hline & \multicolumn{2}{|c|}{ Clase $\mathrm{N}^{\circ} 2$} \\
\hline 1 & $\begin{array}{l}\text { a. Con los elementos dados (cables, pilas, foco } \\
\text { de linterna), diseñe el experimento para } \\
\text { determinar si los materiales suministrados son } \\
\text { conductores o aislantes eléctricos y complete la } \\
\text { tabla } \\
\text { b. Explique el fundamento de la experiencia }\end{array}$ & 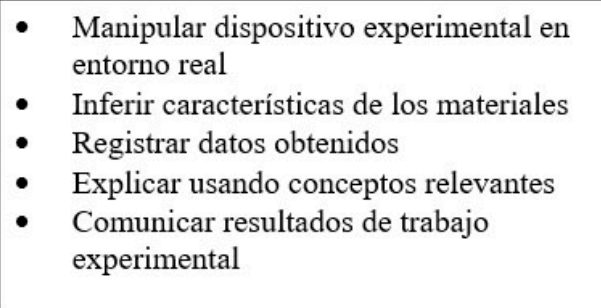 \\
\hline 2 & $\begin{array}{l}\text { Dé tres ejemplos de materiales aislantes y tres } \\
\text { de materiales conductores. }\end{array}$ & - Aplicar conceptos a situaciones nuevas \\
\hline
\end{tabular}




\begin{tabular}{|c|c|c|}
\hline 3 & $\begin{array}{l}\text { a. Por un alambre circulan } 5,6 \times 10^{17} \text { electrones } \\
\text { (carga del electrón } 1,6 \times 10^{-19} \mathrm{C} \text { ) cada } 30 \\
\text { segundos. ¿Cuántos amperes conduce dicho } \\
\text { alambre? } \\
\text { b. Por una sección transversal de alambre pasan } \\
10 \text { coulombios en } 4 \text { segundos. ¿Qué intensidad } \\
\text { tendrá la corriente eléctrica? } \\
\text { c. La intensidad de la corriente eléctrica que } \\
\text { atraviesa un conductor es de } 5 \text { amperios. } \\
\text { ¿Cuántos electrones pasarán por su sección } \\
\text { transversal en } 2 \text { segundos? }\end{array}$ & $\begin{array}{l}\text { - Identificar magnitudes físicas involucradas } \\
\text { - Reconocer las unidades de medida } \\
\text { - Relacionar matemáticamente las magnitudes } \\
\text { correspondientes: electrones, carga, tiempo y } \\
\text { corriente eléctrica y sus unidades de medida. } \\
\text { - Obtener resultados numéricos }\end{array}$ \\
\hline \multicolumn{3}{|c|}{ Clase $\mathrm{N}^{\circ} 3$} \\
\hline 1 & $\begin{array}{l}\text { Para que exista una circulación ordenada de } \\
\text { cargas en un material ¿Qué debe ocurrir? } \\
\text { Muestre en un esquema la situación. } \\
\text { a- ¿Qué es lo que origina la resistencia en un } \\
\text { conductor? } \\
\text { b- ¿cuál es la frase correcta, resistencia de un } \\
\text { material o resistividad de un material? }\end{array}$ & $\begin{array}{l}\text { - Reconocer las condiciones de un circuito. } \\
\text { Diseñar un dispositivo. } \\
\text { - Utilizar la simbología de un circuito y } \\
\text { emplearla correctamente. } \\
\text { - Explicar aplicando el modelo de conducción } \\
\text { eléctrica en términos del rozamiento con la red } \\
\text { cristalina } \\
\text { - Reconocer las variables que afectan a la } \\
\text { resistencia: resistividad, longitud y superficie } \\
\text { Distinguir la propiedad eléctrica de la materia } \\
\text { (resistividad) }\end{array}$ \\
\hline 3 & $\begin{array}{l}\text { Estas porciones de cable son de } \mathrm{Cu} \text { (cobre). } \\
\text { ¿Ambas porciones tendrán la misma } \\
\text { resistencia? ¿Por qué? } \\
\text { ¿Circulará la misma corriente? ¿Por qué? }\end{array}$ & $\begin{array}{l}\text { - Interpretar dibujo. } \\
\text { Vincular las magnitudes: longitud, } \\
\text { resistividad y resistencia. } \\
\text { - Elaborar explicaciones aplicando conceptos } \\
\text { - Interpretar cualitativamente la relación } \\
\text { matemática entre magnitudes importantes } \\
(\mathrm{R}, \mathrm{L} \mathrm{S}, \mathrm{V})\end{array}$ \\
\hline \multicolumn{3}{|c|}{ Clase $N^{\circ}$ 4: LABORATORIO REAL } \\
\hline 1 & $\begin{array}{l}\text { a-Armar el circuito con la fuente de } \\
\text { alimentación, los cables y la resistencia } \\
\text { desconocida } \\
\text { b-Aplicar distintos valores de ddp a una } \\
\text { resistencia R desconocida. } \\
\text { c-Graficar el voltaje (ddp) en función de } \\
\text { corriente ( } \boldsymbol{V} \boldsymbol{v} \boldsymbol{I}) \text {, tomado } 10 \text { pares de valores y } \\
\text { obtener el valor de la resistencia R de la grafica } \\
\text { d- Redactar el informe de la experiencia } \\
\text { realizada donde conste: objetivo, dispositivo } \\
\text { experimental, técnica operatoria, tablas de } \\
\text { valores medidos, tratamiento de datos, cálculo } \\
\text { de errores, resultados. }\end{array}$ & $\begin{array}{l}\text { - } \text { Manipular dispositivos experimentales } \\
\text { - } \text { Diseñar experiencias sencillas } \\
\text { - } \text { Organizar mediciones } \\
\text { - } \quad \text { Trabajar matos } \\
\text { experimentales } \\
\text { - Trazar recta de regresión lineal con Excel } \\
\text { - } \text { Interpretar resultados } \\
\text { - Realizar cálculo de errores } \\
\text { - } \quad \text { Cxplicar procedimientos experimentales } \\
\quad \text { de informes }\end{array}$ \\
\hline
\end{tabular}


$5^{\circ}$ año

Contenidos: Compuestos Orgánicos oxigenados: Ésteres

\section{Objetivos específicos}

- Identificar el grupo funcional de los ésteres.

- Aplicar las reglas de IUPAC para nombrar ésteres.
- Reconocer la presencia de ésteres en productos naturales.

- Identificar propiedades de los ésteres.

- Obtener experimentalmente un producto de importancia industrial como el biodiesel en el laboratorio escolar.

- Identificar ventajas y desventajas del biodiesel como combustible alternativo.

\section{TABLA 3- Compuestos Orgánicos oxigenados: Ésteres}

\begin{tabular}{|c|c|c|}
\hline \multicolumn{2}{|c|}{ CONSIGNAS } & DESEMPEÑOS DE COMPRENSIÓN \\
\hline \multicolumn{3}{|c|}{ Clase $\mathrm{N}^{\circ} 1$ : Formación de Ésteres - Aplicación de reglas de nomenclatura } \\
\hline \multicolumn{2}{|c|}{$\begin{array}{l}\text { 5. A partir de las tarjetas entregadas con fórmulas } \\
\text { semidesarrolladas de diferentes alcoholes y ácidos } \\
\text { carboxílicos, en grupos de } 4 \text { integrantes: } \\
\text { a. proponer la fórmula semidesarrollada del éster formado } \\
\text { en cada caso. } \\
\text { b. escribir la ecuación de esterificación correspondiente. } \\
\text { Ejemplo de tarjetas: }\end{array}$} & \multirow[t]{2}{*}{$\begin{array}{l}\text { Reconocer la formación de un éster a partir } \\
\text { de compuestos orgánicos ya estudiados } \\
\text { (alcoholes y ácidos carboxílicos). } \\
\text { - Identificar el lenguaje icónico - simbólico } \\
\text { propio de la disciplina } \\
\text { - Escribir la fórmula química de un éster. } \\
\text { Formular correctamente una ecuación } \\
\text { química }\end{array}$} \\
\hline $\begin{array}{r}\mathrm{CH}_{3}-\mathrm{CH}_{2}-\mathrm{CH}_{2} \\
\mathrm{OH} \\
\end{array}$ & $\begin{array}{c}\mathrm{CH}_{3}-\mathrm{C}=\mathrm{O} \\
1 \\
\mathrm{OH}\end{array}$ & \\
\hline $\begin{array}{r}\mathrm{CH}_{3}-\mathrm{CH}_{2}-\mathrm{C}=\mathrm{O} \\
1 \\
\mathrm{OH} \\
\end{array}$ & $\mathrm{H}_{3} \mathrm{C}-\mathrm{OH}$ & \\
\hline \multicolumn{2}{|c|}{$\begin{array}{l}\text { 2. A partir de los productos obtenidos en las ecuaciones del } \\
\text { punto } 1 \text { : } \\
\text { a. escribir el nombre de los mismos, siguiendo las reglas de } \\
\text { nomenclatura IUPAC. } \\
\text { b. marcar con un círculo en cada fórmula el grupo } \\
\text { funcional de los ésteres. }\end{array}$} & $\begin{array}{l}\text { - Aplicar reglas de nomenclatura } \\
\text { - Identificar / Reconocer el grupo funcional de } \\
\text { los ésteres }\end{array}$ \\
\hline \multicolumn{3}{|c|}{$\begin{array}{c}\text { Clase } \mathrm{N}^{\circ} 2 \text { : Formulación de ésteres: Presencia de ésteres en la naturaleza } \\
\text { Actividad lúdica: "Ésteres escondidos" }\end{array}$} \\
\hline \multirow{2}{*}{\multicolumn{2}{|c|}{$\begin{array}{l}\text { 1. A partir de la imagen escogida al azar, en grupos de } 4 \\
\text { integrantes: } \\
\text { a. Escribir la fórmula química del éster nombrado junto a la } \\
\text { imagen. } \\
\text { b. Para el éster asignado al grupo, redactar oraciones que } \\
\text { guíen al resto de la clase a deducir nombre, estructura } \\
\text { química y su presencia en el correspondiente producto } \\
\text { natural. } \\
\text { Imágenes a modo de ejemplo: }\end{array}$}} & $\begin{array}{l}\text { - Identificar significados de los prefijos y } \\
\text { sufijos dados en los nombres } \\
\text { - Reconocer los nombres de los alcoholes y } \\
\text { ácidos carboxílicos que dan origen a los } \\
\text { ésteres } \\
\text { - Escribir la fórmula química de un éster } \\
\text { - Emplear en forma adecuada y precisa el } \\
\text { vocabulario propio de la disciplina, en la } \\
\text { expresión oral y escrita. } \\
\text { Reconocer nombre, fórmula química y } \\
\text { ubicación en la naturaleza de un éster. }\end{array}$ \\
\hline & & \\
\hline
\end{tabular}




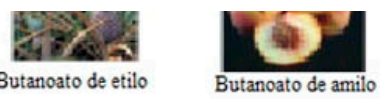

(Fuente de las imágenes: Hipertexto Santillana Química. 2010)

2. Completar el cuadro:

\begin{tabular}{|c|c|c|}
\hline $\begin{array}{c}\text { Nombre } \\
\text { del éster }\end{array}$ & $\begin{array}{c}\text { Fórmula } \\
\text { semidesarrollada }\end{array}$ & $\begin{array}{c}\text { Ubicación en } \\
\text { la naturaleza }\end{array}$ \\
\hline & & \\
\hline
\end{tabular}

- Registrar y organizar información

- Utilizar el lenguaje simbólico propio de la disciplina

\section{Clase $\mathrm{N}^{\circ}$ 3: Propiedades de los ésteres- Aplicaciones comerciales}

1. Explicar la siguiente frase, teniendo en cuenta los datos de la tabla que se adjunta debajo.

Justificar la respuesta.

"Los ésteres son compuestos orgánicos que a diferencia de los ácidos carboxílicos son menos solubles en agua, pero mucho más volátiles que éstos"

(Fuente de texto: Quimica. Saberes clave-Santillana 2011)

(Fuente de datos tabla: Solomons, G.-Química Orgánica. Editorial Limusa -1990)

\begin{tabular}{|c|c|c|c|c|}
\hline $\begin{array}{c}\text { Nombre } \\
\text { del } \\
\text { compuesto }\end{array}$ & $\begin{array}{l}\mathrm{PM} \\
\mathrm{g} / \mathrm{mol}\end{array}$ & $\begin{array}{c}\text { Fórmula } \\
\text { desarrollada }\end{array}$ & $\begin{array}{l}\mathrm{PF} \\
{ }^{\circ} \mathrm{C}\end{array}$ & $\begin{array}{c}\mathrm{PE} \\
{ }^{\circ} \mathrm{C} \text { a } \\
1 \\
\text { atm }\end{array}$ \\
\hline $\begin{array}{l}\text { Ácido } \\
\text { etanoico }\end{array}$ & 60.1 & ${ }_{\mathrm{H}} \mathrm{O}-\mathrm{H}$ & 16,6 & 118 \\
\hline $\begin{array}{l}\text { Etanoato } \\
\text { de etilo }\end{array}$ & 88.1 & 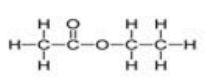 & -84 & 77 \\
\hline
\end{tabular}

\section{Biocombustibles: Biodiesel}

A partir de los siguientes links:

www.biodiesel.com.ar/

www.uaa.mx/investigacion/revista/archivo/revista55/Articulo\%208.pdf

a. Leer comprensivamente la información sobre biodiesel que en ellos se detalla; posteriormente, realizar un esquema donde consigne: -concepto de biodiesel,

-obtención (fuentes),

- reacción de transesterificación,

- ventajas y desventajas de su empleo como combustible.

\section{Clase $N^{\circ}$ 4: Laboratorio: Obtención de Biodiesel- Verificación de su obtención}

1. a. Obtener biodiesel a partir de aceites vegetales usados, empleando los materiales provistos; realizar para ello la secuencia pasos de la técnica operatoria proporcionada.

b. Verificar su obtención mediante la determinación de su densidad, posteriormente compararlos con datos de la bibliografia.

c. Redactar el informe del experimento realizado donde conste: objetivo, materiales, técnica operatoria, observaciones y resultados.
- Interpretar datos contenidos en tabla.

- Comparar propiedades de compuestos orgánicos

- Inferir y generalizar propiedades de compuestos orgánicos de distintas funciones oxigenadas

- Utilizar el lenguaje propio de la disciplina.

- Elaborar justificaciones usando los conceptos científicos adecuados
- Interpretar textos de ciencias

- Registrar y organizar datos

- Identificar la reacción de transesterificación.

- Reconocer / Identificar ventajas y desventajas del empleo de biodiesel respecto a los combustibles tradicionales

- Sintetizar información relevante 


\section{RESULTADOS}

Toda presencia de practicantes en la institución se configura, de alguna manera en una intervención, más aún en el periodo de residencia donde el practicante asume el papel de profesor. Por ello se trata que el trabajo realizado en el terreno áulico sea lo menos intrusivo posible. El período de pasantía participante, permite al residente empatizar con la forma de trabajo en el aula y los lineamientos generales que adopta el profesor a cargo de la cátedra, a quien se le requieren sugerencias para tener en cuenta a la hora de planificar la práctica de residencia. Adoptar este criterio de común acuerdo entre profesor tutor, profesor de la práctica y alumno residente, a la hora de planificar la enseñanza, busca que los estudiantes del secundario se sientan cómodos y puedan trabajar como lo hacen habitualmente con su profesor.

La población escolar donde trabajaron los practicantes estaba constituida por jóvenes de diferentes zonas de la ciudad, incluyendo barrios periféricos, aunque los establecimientos se encuentran en el casco céntrico de la ciudad de Corrientes; pertenecen a familias de muy distintas condiciones económicas, algunos menos favorecidos que otros. La forma de trabajo consistía en hacer todo lo posible dentro del aula, desde la lectura de textos, explicaciones del profesor y realización de actividades de aprendizaje, porque los estudiantes no tenían el hábito de llevar tareas para la casa. Dentro de las recomendaciones de los profesores tutores, fue relevante el hecho de no proponer actividades de mucho tratamiento matemático, ni de alto grado de complejidad. Es por ello que puede verse que las actividades aquí presentadas no pueden considerarse innovadoras y más bien apuntan a conceptos muy básicos pero considerados importantes, dentro del eje temático enseñado.

Las discusiones entre pares y con el docente de práctica en el aula universitaria sirvió como trabajo de campo para recoger información respecto de la labor del residente a la hora de diseñar las clases. Puede decirse que fue una tarea difícil para los residentes, ellos llegaban con la visión de que podrían seleccionar varias actividades de final de capítulos de libros de secundaria y con ello bastaba. Al ponerlos en la situación de desmenuzar la meta de comprensión, ligada a la redacción de los objetivos específicos, y los desempeños de comprensión, ligados a las actividades propuestas, se encontraron con serias dificultades. 'En el caso de Física, la mayor de ellas radicaba en el hecho de tener que ahondar en cada uno de los fenómenos involucrados, donde les resultaba difícil determinar el sistema en estudio y las variables que lo describen, a la vez del comportamiento de las mismas para explicar lo que ocurre. En el caso de química, la dificultad mayor radicó en poder distinguir los desempeños que están involucrados en el uso de la nomenclatura química, tanto para nombrar compuestos como para escribirlos. Algunas expresiones importantes de los estudiantes al momento de analizar lo planificado fueron: "...el análisis de desempeños me bizo pensar bien en el fenómeno", “... ahora tengo claro qué debe hacer el alumno en 
este tema en especial". En ambas disciplinas sí pudieron identificar claramente los desempeños que estaban ligados al quehacer experimental.

\section{CONSIDERACIONES FINALES}

Él diseño de una secuencia didáctica puede entenderse, desde "el punto de vista de la ingeniería didáctica (Artigue, 1992) como un proceso complejo a diferentes niveles: el nivel macro (que garantiza la coherencia de toda la secuencia de enseñanza y aprendizaje) y el nivel micro (de cada sesión)" (Couso, 2011, p69). La coherencia entre los elementos: contenidos, objetivos, actividades, recursos $\mathrm{y}$ formas de evaluar queda establecida al responder claramente las preguntas ¿qué?, ¿para qué?, y ¿cómo enseñar?, incluyendo a la evaluación como parte del proceso de enseñar. Si se focaliza en el nivel micro, se está poniendo la mirada en el clase a clase de la práctica docente y, en ese clase a clase minucioso las actividades que se proponen a los alumnos cobran gran importancia, a la vez que el diseño es lo que más cuesta a los residente.

Como profesores de Física y Química resulta menester definir claramente qué es lo importante que los alumnos aprendan. Haciendo un análisis epistemológico hay que asumir que la Física y la Química explican los fenómenos de la naturaleza a través de modelos construidos por los científicos que tienen diferentes alcances y poder predictivo. El significado de los conceptos físicos y químicos en la secundaria requie- re de vincular el conocimiento directo y la percepción del mundo material con teorías científicas, cuestión que presenta dificultad para los alumnos (Buty y otros, 2004, en Caamaño, 2011). Así entonces, un alumno sabe física o química cuando comprende el modelo científico correspondiente que explica el fenómeno del que trata.

Para enseñar un determinado fenómeno hay que tener bien claro el modelo que explica el mismo y buscar que los desempeños de comprensión guíen el diseño de actividades que permitan desafíos cognitivos, en los cuales se haga uso de los elementos del modelo científico explicativo, por ej. las variables necesarias y sus relaciones, para explicar el comportamiento de un sistema físico o los significados de coeficientes estequiométricos, subíndices de una fórmula o reconocimiento de grupos funcionales, para usar correctamente la nomenclatura química.

Se considera que es importante, para el profesor en formación y los profesores noveles, pensar en profundidad cada contenido que va a enseñar, discriminando el modelo científico puesto en juego. Asumiendo esto como base, es preciso tomar conciencia de qué es lo que se espera que el alumno sea capaz de hacer. Por ello, obligarse a pensar en los desempeños de comprensión que se promueven, de una manera tan minuciosa, como la que se presenta aquí, es sacar a la luz y poner en evidencia cómo vamos a darnos cuenta que el alumno aprende y entiende un determinado contenido. Es una forma de vigilar la coherencia entre contenido, objetivos específicos y actividades de 
una manera más profunda.

Generalmente se piensa que dando una serie de problemas, tipo de final de capítulo de libro, son buenas actividades para aprender, dado que quien resuelve autónomamente un problema es alguien que aplica los conceptos a la situación planteada y, por ende, comprende o aprende significativamente. En el caso de los estudiantes de secundaria, es importante ponerlos en situación de observar cognitivamente, más allá de lo que ven los ojos, registrar datos cualitativos o medidos con instrumentos, procesar datos (con cálculos y trazados de gráficas), analizar resultados, relacionar magnitudes descriptoras de un fenómeno, usar la nomenclatura química (hablar y escribir "en química), dar explicaciones, elaborar justificaciones aplicando conceptos relevantes, todos desempeños que hacen a la forma de generar el conocimiento científico.

Hay formar profesores que entiendan bien la ciencia que enseñan, se apasionen por ella y generen buenas actividades para sus alumnos, buscando terminar con las generaciones de estudiantes adolescentes que huyen del estudio de la Física y la Química. Sólo así se podrán despertar más vocaciones científico-tecnológicas.

\section{REFERENCIAS}

Buty, C. y otros (2004). Learning hyphoteses and associated tool to design and to analyse teaching-learning sequences. International Journal of Science Education, 26(5), citado en Caamaño. A. (2011). Didáctica de la Física y la Química. Barcelona, España: Graó.

Couso, D. (2011). Las secuencias didácticas em la enseñanza y el aprendizaje de lãs ciências: modelos para su diseño y validación, en Caamaño. A. (2011). Didáctica de la Física y la Química. Capítulo 3.Barcelona, España: Graó.

Davini, M.A. (2015). La formación en la práctica docente. Buenos Aires: Paidós. Perkins, D. (1999). ¿Qué es la comprensión? En
Stone Wiske,M (Comp.), La enseñanza para la comprensión. Vinculación entre la investigación y la práctica. Buenos Aires: Paidós. Sanjurjo, L. y Foresi, Ma. F. (2015). La enseñanza como preocupación teórica de la didáctica y como preocupación teóricopráctica de los profesores. En Massa, M y otros (Eds.), La enseñanza de las Ciencias Naturales en la Escuela Media. Fundamentos y desafíos. Rosario: Homo Sapiens. Sanjurjo, L. O. y otros (2004). La residencia docente: un espacio para la práctica reflexiva. https://es.scribd.com/document/230398601/La-Residencia-Docente. Sitio consultado en septiembre de 2016. 